\title{
Exploring self-management within hospital-based stroke care: current and future opportunities.
}

Purpose: This study explored stroke self-management within a tertiary hospital setting from the perspectives of health professionals working across the continuum of stroke care.

Materials and Methods: A qualitative descriptive design guided five focus groups in the acute stroke service $(n=2)$, inpatient rehabilitation $(n=2)$, and outpatient day hospital service $(n=1)$. Focus groups were transcribed verbatim and analysed using thematic analysis.

Results: Twenty-eight health professionals participated representing medical, nursing, and allied health services. Two themes emerged from the data: Pieces of a puzzle illustrates the inconsistent understanding about self-management with elements of the puzzle described but rarely within the full concept of self-management; Readiness for self-management highlighted that although self-management should commence in the acute setting, there were many factors influencing why this was not always happening.

Conclusions: A consistent conceptualisation and approach to stroke self-management in the hospital setting is required. Interprofessional education and shared intentional language can enhance understanding and practice.

Keywords: multidisciplinary team; acute setting; rehabilitation; focus groups; phenomenology 


\section{Introduction}

There is a growing interest in the role of self-management support in stroke care [1-4] however it remains unclear how health services can best support self-management in stroke survivors [5]. This is an important area for research focus as the burden on health services may reduce when stroke survivors are empowered to become effective self-managers of their condition [6,7]. Literature reports that self-management support builds a person's confidence to identify and address the problems that accompany their condition $[6,8,9]$. Health professionals coach individuals to develop the skills and attitudes required to set goals, action plan, problem solve, access appropriate resources, develop partnerships with health professionals, and make decisions [8]. People who become good self-managers demonstrate sufficient self-efficacy to apply these skills in medical, emotional, and role self-management behaviours [3].

Stroke specific self-management support tailor these skills to be more relevant and applicable to stroke [10] and has been explored primarily in community settings as discrete programs that teach people self-management skills $[1,4]$. Systematic reviews demonstrate preliminary evidence of benefit for participants, specifically in outcomes for quality of life $[1,4]$, self-efficacy [4], disability, confidence in recovery, and some physical measures [1]. However, confidence in these conclusions is limited by the variability in the quality of the research, as well as the heterogeneity in program content and delivery $[1,4]$.

People with stroke report an unmet need for education [11] and self-management skills following discharge from health services [12], pointing to a need for self-management support during hospital care [13]. However, hospital-based promotion of stroke self-management has been reported to a lesser extent than in the community, resulting in a limited understanding of its feasibility or optimal format $[14,15]$. One acute stroke team received interprofessional education to embed self-management practices in daily care and demonstrated more collaborative practice [15]. Another team, from an inpatient rehabilitation unit, found that a self-management approach 
promoting independent physical activity increased patient engagement in exercises external to therapy [14]. Importantly, the impact of any self-management support relies on health professionals who empower, partner with, and offer resources to individuals [16]. In contrast, when health professionals are reluctant to relinquish control over planning [17] or focus on achieving the agenda of the health setting [18], limited self-management support is achieved. These findings highlight how important it is for the multidisciplinary team to understand selfmanagement concepts in order to achieve self-management outcomes for patients $[12,14,19]$. Given self-management within the community has been extensively explored further investigation into self-management understanding and practices in a hospital setting is warranted. The purpose of this study is to explore the multidisciplinary team's understanding of selfmanagement and opportunities they identified for its application across the stroke continuum in a tertiary hospital.

\section{Methods}

\section{Research design}

A qualitative approach supported exploration of clinicians' experiences of promoting stroke selfmanagement within a hospital setting. Qualitative description [20] guided the study methods supporting an exploration of the phenomenon of stroke self-management from the perspectives of the health professionals with a relativist ontology and subjectivist epistemology. Ethical approval was gained from [removed for submission] and [removed for submission].

\section{Study Setting,}

The study was conducted in a tertiary hospital where the stroke continuum of care encompasses the acute stroke unit, inpatient rehabilitation unit, and outpatient day hospital clinic. There is no articulated mission for stroke care that refers to a self-management approach; however, elements of stroke self-management support are integrated into standard care. For example, acute stroke 
patients receive a booklet produced by the [blinded for submission] that provides basic stroke education, encourages goal setting, and directs people to online and phone supports. People with stroke and their carers in the rehabilitation unit are invited to fortnightly, multidisciplinary education sessions that draw on the experiences of a stroke survivor volunteer to discuss the content of this booklet in more detail.

\section{Participants}

Convenience sampling recruited participants from the multidisciplinary teams of the three stroke care units. There were no other specific inclusion or exclusion criteria. Potential participants received an email invitation that provided details of the study, including the dates and locations of the focus groups.

\section{Data collection procedure}

To reduce the risk of coercion, the researcher responsible for recruiting participants $(\mathrm{KB})$ was not involved in gaining consent, participating in, or conducting the focus groups [21]. All participants voluntarily attended and provided written consent prior to involvement in the focus group. Five focus groups were facilitated by LG and AG, ranging in length from 35 to 50 minutes, and attended by a minimum of four and maximum of seven participants. Two separate focus groups were conducted with the acute stroke and inpatient rehabilitation teams. One focus group was conducted with the rehabilitation day hospital team due to the smaller number of clinicians. The focus group enriched the data as participants' interactions allowed for reflection and clarification of understanding [22,23]. An interview guide offered structure to explore participants' understanding of self-management, examples of practices promoting selfmanagement, and contextual influences, yet provided enough flexibility to explore topics generated by participants [24]. The following definition was introduced in the early section of the focus group to orientate the participants - self-management is the demonstration of 
behaviours such as problem solving, action planning, decision making, etc that support the individual to manage the changing life roles, and emotional and physical effects of the chronic condition [3]. The focus group questions explored the self-management support delivered across the continuum of care and within the specific work unit and then focussed specifically on the self-management behaviours considered essential for stroke survivors; how this was supported, where in the care continuum and by who; what was their role in supporting self-management behaviours; and what could be developed further. Focus groups were recorded for transcription and brief field notes were taken to understand the data during analysis [24].

\section{Data analysis}

Three researchers (AG, LG, KB) read through all the transcripts to familiarise themselves with the data prior to commencing analysis. Inductive thematic analysis [20] occurred after the completion of all focus groups. The researchers independently coded transcripts and met to discuss and identify patterns in the codes. The patterns informed the development of emergent themes. The emergent themes were mapped against the transcripts and further refined to ensure the findings were reflective of the data [25]. Four preliminary themes were developed and described, and further discussions identified patterns that supported their integration as subthemes within the final two themes presented here. The summaries of the final themes were finalised and sent via email to participants for member checking [26,27]. The participants were asked to reply with any changes or clarifications. No responses were received. The primary researcher engaged in a process of reflexivity, recording assumptions and developing perspectives in a research journal [28] to discuss during research team meetings.

\section{Rigor and trustworthiness}

The primary researcher engaged in a process of reflexivity, recording assumptions and developing perspectives in a research journal [28] to discuss during research team meetings. LG, 
$\mathrm{KW}$ and $\mathrm{KB}$ all have experience in stroke rehabilitation and bracketing of assumptions occurred prior, during, and after the data collection and analysis processes. Thus, open discussions and peer debriefing within the research team were important to ensure that the findings were representative of the data. Member checking included presentation of summaries of the themes to the participants and was conducted as described in the data analysis section. Triangulation of the data within and across the three different teams allowed for the development of a more comprehensive finding.

\section{Results}

There were 28 participants recruited with a total of five clinicians from day hospital (average years in ward $=7.2$ ), thirteen from inpatient rehabilitation (average years in ward $=4.88$ ) and ten from the acute stroke unit (average years in ward $=1.44$ ). Participating professional groups included nurses, physiotherapists, speech pathologists, neuropsychologists, occupational therapists, dieticians, social workers, and medical staff. All focus groups included representatives from nursing and allied health; however, only the day hospital focus group included a member of medical staff. The years of experience in stroke care have been averaged for allied health (4.2 years) and nursing staff ( 8.75 years). After each quote the participant's years of experience is indicated as either greater than or less than these figures. For participant demographics refer to table 1 .

Insert table 1 about here.

The two overarching themes were: Pieces of the puzzleós] and Readiness for selfmanagement. Pieces of the puzzle described the absence of a unified understanding and approach to providing self-management support across the teams and settings. This led to various descriptions, understandings and applications of the concept that were influenced by clinicians' 
professional paradigm and area of practice. Readiness for self-management demonstrated the various opinions within the teams regarding when self-management support should commence, and the individual and institutional factors that influence its application in a hospital setting.

\section{Pieces of the Puzzle}

The overarching representation from the participants was one of separate but interrelated puzzle pieces scattered within and across the teams, with a need for a common thread or picture to be developed and encompass the entire concept of self-management. The participants had different ways that they understood self-management, differing applications, and this was context-specific across the three services and professions.

Differing conceptualisations: Clinicians articulated different definitions of self-management that included “...being able to identify goals...” (Allied Health $<4.2$ years), “...the ability to be able to advocate for themselves..." (Allied Health $<4.2$ years), or to, “...carry through with exercises..." (Allied Health $<4.2$ years). In doing so, the participants were naming key behaviours of self-management but not able to readily identify a definition to describe the concept. In fact, $\mathrm{t}$ he term self-management was rarely used: "I don't tend to use the phrase self-management as much just the word independence." (Allied Health $>4.2$ years). This conveyed the impression that there was no shared conceptualisation or language to articulate the concept of self-management as a whole.

Application - Self-management support was discussed by several participants. Education and information provision were identified by several participants as a key component. General stroke education often commenced in the acute setting, focusing on the individual's physical capacity, function and risk of complications, "I find that my early education is around just preventing bad things from happening, secondary changes..." (Allied Health $<4.2$ years). Education was also used to support ongoing care, “...depending on the patient, we have 
to give some of our education strategies early, ... particularly from a communication or swallowing perspective... because that obviously impacts their care..." (Allied Health $>4.2$ years). Clinicians in the acute setting utilised educational resources, “...we have these My Stroke Journey packages that we also give them,... that sort of helps them understand some of their risk factors..." (Nursing $>8.75$ years). This booklet was shared with family members to increase their understanding of stroke, however it was not often referred to by participants from rehabilitation settings. Inpatient rehabilitation clinicians spoke of a stroke education group to help patients to build their knowledge of stroke, staff perceived that the focus of the stroke education group was “....on basic function and discharge planning, but not necessarily as much education, when managing a chronic condition." (Allied Health $>4.2$ years).

Goal setting was recognised as another important element of self-management support, "some of our goal setting process ... is about well what's important to you what do you want to do so it is, I suppose at some level like some control back to that person... "'(Allied Health $<4.2$ years). It was recognised as a strategy that supported the active involvement of the individual in treatment planning, “...if you've had a really nice goal-setting session with someone, um, when they come into the gym, you can say to them, what do you want to work on today?" (Allied Health $<4.2$ years). Goal setting appeared to be the key strategy for empowering the individual.

Context-specific - Conceptualisation of self-management and the priority placed on it varied across the different clinical settings. At the beginning of the focus group some participants in the inpatient settings held a narrower perspective of what self-management involved, often reflective of each participant's unique professional paradigm. For example, nursing staff described selfmanagement as independence in self-care, physiotherapists described engagement in exercises and speech pathologists described improving communication. However, the simple act of participating in the focus group served as an opportunity to develop their understanding of self- 
management, and how clinicians can provide self-management support. For example, a participant who at the beginning of the group described self-management as functional independence had by the end of the group progressed to questioning, "So how to get ... staff to have those sort of knowledge and skills to engage with the patient, to encourage them to participate in those self-management skills." (Nursing $<8.75$ years). Another participant recognised that there should be an increased focus on empowering the individual and providing more choice.

The participants in the day hospital focus group appeared to be more readily able to put the puzzle of stroke self-management together, as reflected by this comment:

I think [self-management] is probably everything isn't it?...., how to adapt and about whether they have got a new disability... things change, about work about how they see their role even about umm lifestyle changes with that as well, medication, diet. (Nursing $>8.75$ years).

They discussed seeing the completed puzzle, which was supporting individuals to manage life in their home and community with their new condition as opposed to focusing on puzzle pieces of self-management. The change of context to the community provided opportunities for the promotion of self-management to be contextualised, and for rehabilitation to focus on a broader scope of previously held roles. This finding supported discussions within the other groups regarding the best time to support the development of skills for self-management as is illustrated in the next theme.

\section{Readiness for self-management}

Like the puzzle pieces of self-management, the teams presented interrelated ideas about when patients were ready to receive support for self-management. These opinions primarily related to perceived barriers encountered at the time of acute management, the timing of hospital 
discharge, and when a patient or family displayed 'readiness' to engage in self-management behaviours.

The influence of context: The rehabilitation clinicians suggested that self-management could, and perhaps should, start in the acute setting. "I think it should start right at the start and I do think... it should get reinforced throughout their journey." (Allied Health $>4.2$ years). While some acute clinicians agreed that self-management should commence "...right from the beginning for ... the majority of the patients”, (Nursing $>8.75$ years) they also described barriers such as the rate of patients' recovery, resourcing, and time availability. Acute clinicians reported difficulty planning what they would be working on given that an individual's presentation had the propensity to change quickly:

... they change quickly as well ... and then you have to go and see them again. Which, I guess, makes me think about, ... what self-management strategies ... or what information am I giving and what's my plan for this patient... (Allied Health $<4.2$ years).

For people transferring to rehabilitation, acute clinicians would focus on completing assessments and considered that self-management would occur during rehabilitation, “...our role is to get them to somewhere where they can engage in that self-management." (Allied Health $<4.2$ years). The transitional nature and short time frame of the acute setting led to the suggestion that commencement of self-management could be detrimental: “...I ...make the decision ... it's not appropriate for me to build a quick relationship and try and help these people when, ... they can receive long term follow up in rehab or in another hospital." (Allied Health >4.2 years).

When hospital discharge occurred from the acute setting, clinicians offered "information about ways in which they can self-manage post-discharge ... in the acute setting so that they can apply that once they're returning home." (Allied Health $<4.2$ years). However, there was no consistent approach or follow-up described to achieve this. An upcoming discharge from inpatient rehabilitation was also seen as a key trigger for promoting self-management. A 
rehabilitation clinician commented, "I find that the transition for most people seems quite natural. I think there are some people, where I guess, you ... need to start drumming in the selfmanagement, because you know their discharge date..." (Allied Health $<4.2$ years). Within the day hospital group, it was unanimous that self-management often became more applicable after discharge to home and as individuals were required to manage life and their previously held roles.

Personal factors: Individuals' readiness for self-management was often described in relation to physical or cognitive recovery, “...If ... the damage to the brain is very severe and then, they cannot really use their limbs at all, there'll be no hope for them to self-management [sic] in any way." (Nursing $<8.75$ years). An individual's awareness of their condition was also seen as a factor impacting self-management. "I think they need to have a level of insight ..., to be able to take and run with their self-management...., and that will differ for patients, depending on where they are in their journey." (Allied Health $<4.2$ years). Similarly, clinicians noticed individuals who were actively involved in their therapy, stating that this was often an indication that it was an ideal time to introduce self-management support, "If they start asking a lot of questions... so they are really keen to learn, keen to engage." (Allied Health $<4.2$ years). This was reinforced by the observation that they "...had a lot of success with people that are naturally driven..." (Allied Health $<4.2$ years) suggesting clinicians perceived individuals who were motivated or engaged in their recovery were more likely to respond to self-management support.

The willingness of families to be engaged with the therapy process and promote selfmanagement behaviours seemed to influence decisions about when to commence support for self-management. Engaged families would motivate the individual, gather information on their behalf, and continue therapy programmes in the clinician's absence.

...the people... I've seen in day hospital that have really ... taken on ...some activities around managing their recovery, have not had significant cognitive issues ... or had a really 
supportive family member or carer that's attended their therapy and has kind of partnered in that with them. (Allied Health $>4.2$ years).

In contrast, clinicians identified times when family characteristics were not consistent with promoting self-management. Families could hinder self-management skill development because, “...the carer sometimes won't let someone self-manage...” (Nursing $>8.75$ years) and “...selfmanagement in those early stages is not letting the family members take over care for the patient...” (Nursing $<8.75$ years).

\section{Discussion}

Stroke self-management support has previously been explored in the context of the multidisciplinary team in the community $[17,29]$ and this study extended that work to explore the perspectives of clinicians working in multidisciplinary teams across hospital-based care. The findings suggest that support for self-management across the care continuum was impacted by an inconsistent understanding of self-management within and across teams, and differing expectations and beliefs about readiness for self-management support.

Inconsistent language was used to describe self-management, perhaps influenced by the limited self-management terminology available in current stroke literature $[3,30]$. However, the clinicians identified that they purposively avoided the term self-management, opting for what they considered more client-friendly terminology such as independence or education. This is in contrast to recommendations that clinicians should be intentional in the language they adopt to support a shared understanding and recognition of self-management [31]. While clients are reportedly unfamiliar with the term self-management, studies demonstrate that they can develop their own definition, typically describing it as a strategy to support independence in their life $[12,32]$. This reinforces that clinicians should not necessarily avoid the term self-management, rather it should be incorporated into their existing vernacular and explained in conjunction with 
other empowering language. The finding suggests an opportunity for the teams to develop shared terminology that may in turn, facilitate more self-management support in a hospital-based setting.

Few clinicians in this study could articulate the whole concept of self-management, instead describing elements such as resource utilisation, goal setting and education [8]. Clinicians within the inpatient units articulated skills that appeared to support medical selfmanagement as outlined by Parke et al. [3], such as performing exercises and increasing knowledge of the condition. However, there was limited discussion of the other core skills including decision making, problem solving and forming partnerships with clinicians, suggesting that role and emotional self-management behaviours were considered less often [3]. For individuals to receive comprehensive support in developing self-management skills and behaviours, clinicians first need a strong foundational knowledge of all self-management principles [29]. Day hospital clinicians were the only participant group to address all elements of self-management in their practice, perhaps due to working with clients who had returned home and were better able to clearly articulate their needs [17]. Overall, it was clear that different understandings of self-management within and across the teams impacted practice. This highlights opportunities to develop shared knowledge and skills, and consistent approach across the stroke continuum of care.

The second theme identified various beliefs about readiness for self-management, with clinicians suggesting that this varied significantly between individuals. It has previously been demonstrated that clients and clinicians alike report differing ideas regarding the best time to commence [29,33-35] and that a standardised approach is considered to be ineffective due to the individualised nature of self-management [33]. Some individuals may demonstrate readiness to engage in self-management soon after stroke and evidence demonstrates that self-management support at this stage is feasible $[14,15]$. However, there is also concern that some individuals 
may not be ready, and there are mixed opinions from multidisciplinary teams regarding how realistic self-management is within the acute setting, given the barriers of time and resourcing [17].

All clinicians, regardless of the setting, agreed there was a need to support selfmanagement prior to discharge, but could not agree when this should commence. The acute care clinicians prioritised patients who were discharged directly to home and suggested that the rehabilitation clinicians would commence self-management with the patients transferring to their units. On returning home, individuals are responsible for managing the symptoms of their condition [5], often with reduced contact from health professionals [36]. Perhaps aware of this, clinicians in this study highlighted the value of promoting self-management, usually in the form of education, prior to discharge. Whilst this strategy for developing self-management is beneficial, education alone is not sufficient to promote self-efficacy and support behavioural change [29]. Parke and colleagues [3] describe a process that includes the acquisition of selfmanagement skills followed by the independent incorporation of these skills into selfmanagement behaviours, thereby building individuals' self-efficacy.

The findings suggest that family involvement in self-management is frequently identified as a strategy to improve an individual's engagement; however, families may become overly involved and limit opportunities for patients to practise self-management behaviours [14,30,32,37-39]. Families who are willing to support self-management outside of the therapistdriven sessions provide greater opportunity for patients to master self-management skills within the hospital setting [40], which is particularly valuable for time-poor clinicians [17]. Many families articulate their need for skill development in this area [40] indicating that they should be included for a successful approach to self-management support.

The individual's willingness and motivation to engage is often a key factor influencing clinicians' decisions about whether or not to provide self-management $[41,42]$. Clinicians 
highlighted that individuals who appeared motivated were given more self-management support. However, individuals who experience low self-efficacy in self-management skills [43] may take small steps during hospitalisation to better facilitate self-management behaviours after discharge $[44,45]$. Indeed, people who receive self-management input from clinicians typically have higher engagement in their health care [46], highlighting the need for the clinicians to include all individuals regardless of level of motivation. Clinicians in this study also indicated that a level of physical or cognitive recovery influenced whether self-management training was provided. This belief has been described elsewhere [35]; however, may result in clinicians further withholding support from individuals who they feel are unable to engage [19,32,35].

Finally, for some participants engagement in the focus groups led to an evolving conceptualisation of self-management . The participation in a focus group allowed perspectives to be clarified, justified, and refined in light of the discussions and perspectives from other participants [47]. This outcome demonstrated a readiness to listen and grow in understanding that augers well for knowledge and service development. It appeared at times as if the focus group served as a community of practice, providing a space for discussion and development of understanding. This may be one approach that could be taken to foster the development of a consistent understanding of and approach to self-management support across the three services.

This study has demonstrated the need for further collaborative training to foster a shared understanding and application of self-management. Interdisciplinary training has shown to led to improved communication of individuals' goals, an increase in self-management provision and consistent application of self-management across the team $[31,48]$. However, staff training alone is not indicative of practice change [29,39] rather, a transformative approach to practice is required, beginning with clinicians first acknowledging their perspectives and methods of practice to consider how these influence self-management support $[5,39]$. 


\section{Limitations and future research}

This study included multidisciplinary teams within a single organisation which limits the generalisation of findings to other tertiary hospitals. Although a broad representation of health disciplines was achieved across the groups, there was only one dietician and one doctor recruited. The absence of doctors, in particular, may explain why there was a stronger focus on exercise and education as elements of self-management, and less discussion on managing stroke risk factors in the focus groups. Limited participation from doctors has been reported elsewhere [15], but their significant involvement in patient care means their presence in these studies is essential. Practice change to support self-management implementation is needed, with this study demonstrating that further investigation into practice guidelines to support this change is required. This study identifies a number of avenues for future research including development of interdisciplinary training in self-management and exploration of strategies to support teams to integrate self-management development into current hospital-based services.

\section{Conclusion}

Self-management in stroke care is a multifactorial approach which lacks clarity, consensus and consistency within inpatient hospital settings. However, with a growing body of literature highlighting the importance of self-management support across the stroke continuum $[3,4,13,14]$ it is necessary to establish a consistent understanding. The lack of a common conceptualisation, language and approach - particularly in relation to timing - both at a local level and more globally, impacts on application in practice and research. This study has reinforced the need for strategies to enhance implementation in a hospital setting, including interprofessional training $[29,31,39,41,48]$; adopting an agreed shared language [31]; and staff reflection on their conceptualisations and practice to facilitate service transformation [5,39]. 


\section{Acknowledgements}

The author would like to acknowledge the contribution of all the clinicians who gave their time to participate in this study.

Declaration of Interests

The authors report no conflict of interest. 


\section{Reference}

1. Lennon S, McKenna S, Jones F. Self-management programmes for people post stroke: A systematic review. Clin Rehabil. 2013;27(10):867-878.

2. Whitehead L. Self-management programmes for quality of life in people with stroke. International J Nurs Pract. 2018;24(2):e12612-n/a.

3. Parke HL, Epiphaniou E, Pearce G, et al. Self-management support interventions for stroke survivors: A systematic meta-review. PLoS ONE. 2015;10(7):e0131448.

4. Fryer CE, Luker JA, McDonnell MN, et al. Self management programmes for quality of life in people with stroke. Cochrane Database of Systematic Reviews. 2016 (8).

5. Mikkonen I, Hynynen M-A. Health care professionals' views about supporting patients' self-management. Health Educ. 2012;112(5):396-405.

6. Grady PA, Gough LL. Self-management: A comprehensive approach to management of chronic conditions. Am J Public Health. 2014;104(8):e25-e31.

7. Barlow J, Wright C, Sheasby J, et al. Self-management approaches for people with chronic conditions: A review. Patient Educ Couns. 2002;48.

8. Lorig KR, Holman HR. Self-management education: History, definition, outcomes, and mechanisms. Ann Behav Med. 2003;26(1):1-7.

9. Barrecheguren M, Bourbeau J. Self-management strategies in chronic obstructive pulmonary disease: A first step toward personalized medicine. Curr OpinPulm Med. 2018;24(2):191-198.

10. Jones F. Strategies to enhance chronic disease self-management: How can we apply this to stroke? Disabil Rehabil. 2006;28(13-14):841-847.

11. McKevitt C, Fudge N, Redfern J, et al. Self-reported long-term needs after stroke. Stroke. 2011;42(5):1398-1403.

12. Boger EJ, Demain SH, Latter SM. Stroke self-management: A focus group study to identify the factors influencing self-management following stroke. Int J Nurs. 2015;52(1):175-187.

13. Jones F, McKevitt C, Riazi A, et al. How is rehabilitation with and without an integrated self-management approach perceived by UK community-dwelling stroke survivors? A qualitative process evaluation to explore implementation and contextual variations. BMJ Open. 2017;7(4):e014109.

14. DePaul VG, Bosch J, Oczkowski WJ, et al. Promoting Independent Mobility-related Physical ACTivity (IMPACT) in an inpatient stroke rehabilitation unit: A proof-ofconcept evaluation of self-management intervention. Disabil Rehabil. 2019:1-10.

15. Mäkelä P, Gawned S, Jones F. Starting early: Integration of self-management support services BMJ Quality Improvement Reports 2014:1-7.

16. Ndemera H, Bhengu B. Motivators and barriers to self-management among kidney transplant recipients in selected state hospitals in South Africa: A qualitative study. Health Sc J. 2017;11(5):1-13.

17. Norris M, Kilbride C. From dictatorship to a reluctant democracy: Stroke therapists talking about self-management. Disabil Rehabil. 2014 2014/01/01;36(1):32-38. 
18. Levack WMM, Dean SG, Siegert RJ, et al. Navigating patient-centered goal setting in inpatient stroke rehabilitation: How clinicians control the process to meet perceived professional responsibilities. Patient Educ Couns. 2011;85(2):206-213.

19. McKenna S, Jones F, Glenfield P, et al. Bridges self-management program for people with stroke in the community: A feasibility randomized controlled trial. Int J Stroke. 2015;10(5):697-704.

20. Sandelowski M. Whatever happened to qualitative description? Res Nurs Health. 2000;23: 334-340.

21. Lawrence Neuman W. Social Research Methods: Qualitative and quantitative approaches. 7, editor. Boston, MA: Pearson Education, Inc.; 2011.

22. Côté-Arsenault D, Morrison-Beedy D. Women's voices reflecting changed expectations for pregnancy after perinatal loss. J Nurs Scholarship. 2001;33(3):239-244.

23. Bradbury-Jones C, Sambrook S, Irvine F. The phenomenological focus group: An oxymoron? J Adv Nurs. 2009;65(3):663-671.

24. Chadwick B, Gill P, Stewart K, et al. Methods of data collection in qualitative research: Interviews and focus groups. BDJ. 2008;204(6):291-295.

25. Braun V, Clarke V. Using thematic analysis in psychology. Qual Res Psychol. 2006;3(2):77-101.

26. Bradbury-Jones C, Irvine F, Sambrook S. Phenomenology and participant feedback: Convention or contention? Nurs Res. 2010;17(2):25-33.

27. Sandelowski M. Reembodying qualitative inquiry. In: Atkinson P, Delamont S, editors. Sage qualitative research methods Vol. 12. Thousand Oaks: SAGE Publications, Inc; 2010. p. 104-115.

28. Guba EG, Lincoln YS. Competing paradigms in qualitative research. In: Denzin NK, Lincoln YS, editors. Handbook of Qualitative Research Thousand Oaks, CA: SAGE Publication, Inc; 1994. p. 105-117.

29. Taylor A, Monsanto X, Kilgour H, et al. Attitudes of healthcare professionals towards self-management for patients with stroke. N Z J Physiother. 2019;47(1):25.

30. Pearce G, Pinnock H, Epiphaniou E, et al. Experiences of self-management support following a stroke: A meta-review of qualitative systematic reviews. PLOS ONE. 2015;10(12):e0141803.

31. Kulnik ST, Pöstges H, Brimicombe L, et al. Implementing an interprofessional model of self-management support across a community workforce: A mixed-methods evaluation study. J Interprof Care. 2017;31(1):75-84.

32. Sadler E, Wolfe CDA, Jones F, et al. Exploring stroke survivors' and physiotherapists' views of self-management after stroke: A qualitative study in the UK. BMJ Open. 2017;7(3).

33. Clark E, Bennett K, Ward N, et al. One size does not fit all - Stroke survivor's views on group self-management interventions. Disabil Rehabil 2018;40(5):569-576.

34. Singer B, Jones F, Lennon S. Adapting the Bridges stroke self-management program for use in Australia. Int J Ther Rehabil. 2018;25(8):414-423. 
35. Satink T, Cup E, de Swart B, et al. Self-management: Challenges for allied healthcare professionals in stroke rehabilitation - a focus group study. Disabil Rehabil. 2015;37(19):1745-1752.

36. de Silva D. Evidence: Helping people help themselves: A review of the evidence considering whether it is worthwhile to support self-management. Health Foundation website: The Evidence Centre; 2011.

37. Sørensen SL, Pedersen SKS, Pallesen H. Social psychological mechanisms and processes in a novel, health professional-led, self-management intervention for older stroke individuals: A synthesis and phenomenological study. BMC Health Services Res. 2019 2019/05/22;19(1):320.

38. Norris M, Jones F, Kilbride C, et al. Exploring the experience of facilitating selfmanagement with minority ethnic stroke survivors: A qualitative study of therapists' perceptions. Disabil Rehabil. 2014;36(26):2252-2261.

39. Davies F, Wood F, Bullock A, et al. Shifting mindsets: A realist synthesis of evidence from self-management support training. Medic Educ. 2018;52(3):274-287.

40. Bower K, Gustafsson L, Hoffmann T, et al. Self-management of upper limb recovery after stroke: How effectively do occupational therapists and physiotherapists train clients and carers? Brit J Occup Ther. 2012;75(4):180-187.

41. Mudge S, Kayes N, McPherson K. Who is in control? Clinicians' view on their role in self-management approaches: A qualitative metasynthesis. BMJ Open. 2015;5(5):e007413-e007413.

42. Bos-Touwen ID, Trappenburg JCA, Van Der Wulp I, et al. Patient factors that influence clinicians' decision making in self-management support : A clinical vignette study. PLoS ONE. 2017;12(2):e0171251.

43. Damush TM, Ofner S, Yu Z, et al. Implementation of a stroke self-management program: A randomized controlled pilot study of veterans with stroke: A randomized controlled pilot study of veterans with stroke [Article]. Transl Behav Med. 2011;1(4):561-572.

44. Pollack AH, Backonja U, Miller AD, et al., editors. Closing the gap: Supporting patients' transition to self-management after hospitalization. Conference on Human Factors in Computer Science 2016; California

45. Sit J, Chair SY, Choi K, et al. Do empowered stroke patients perform better at selfmanagement and functional recovery after a stroke? A randomized controlled trial. Clin Interv Aging. 2016 10/01; Volume 11:1441-1450.

46. Hibbard J, Cunningham P. How engaged are consumers in their health and health care, and why does it matter? Research Brief. 2008 11/01;8:1-9.

47. Wilkinson S. Focus groups in health research: Exploring the meanings of health and illness. J Health Psychol. 1998;3(3):329-348.

48. Jones F, Waters C, Benson L, et al. Evaluation of a shared approach to interprofessional learning about stroke self-management. J Interprof Care. 2012;26(6):514-516. 
Table 1. Participant demographics

\begin{tabular}{|l|l|l|l|}
\hline Profession & Number & $\begin{array}{l}\text { Yrs. experience } \\
\text { average (min-max) }\end{array}$ & $\begin{array}{l}\text { Yrs. in stroke care } \\
\text { average (min-max) }\end{array}$ \\
\hline Doctor & 1 & 38 & 33 \\
Nurse & 10 & $19.85(1.5-40)$ & $8.7(1.5-23)$ \\
Occupational Therapist & 5 & $6.7(0.5-15)$ & $2.5(0.5-7)$ \\
Physiotherapists & 3 & $7(5-10)$ & $3.83(2.5-5)$ \\
Speech Pathologist & 4 & $10.5(2-25)$ & $8.75(1.5-25)$ \\
Social Work & 2 & $8.75(2.5-15)$ & $1.34(0.67-2)$ \\
Dietician & 1 & 13 & $\sim 13$ \\
Neuropsychologist & 2 & $20(20-20)$ & $1(1-1)$ \\
\hline
\end{tabular}

